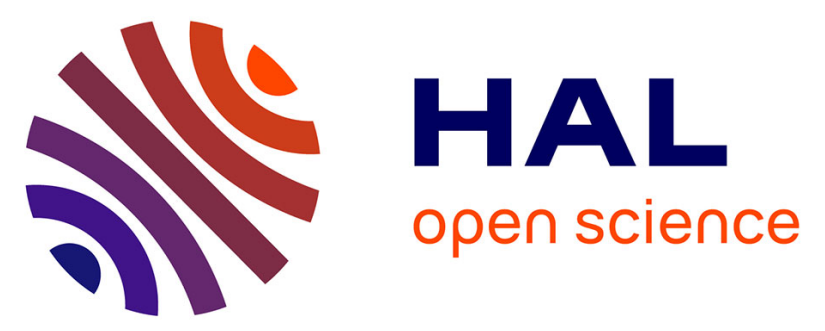

\title{
Towards a better understanding of wood cell wall characterisation with contact resonance atomic force microscopy
}

Olivier Arnould, Richard Arinero

\section{- To cite this version:}

Olivier Arnould, Richard Arinero. Towards a better understanding of wood cell wall characterisation with contact resonance atomic force microscopy. Composites Part A: Applied Science and Manufacturing, 2015, 74, pp.69-76. 10.1016/j.compositesa.2015.03.026 . hal-01232551

\section{HAL Id: hal-01232551 \\ https://hal.science/hal-01232551}

Submitted on 23 Nov 2015

HAL is a multi-disciplinary open access archive for the deposit and dissemination of scientific research documents, whether they are published or not. The documents may come from teaching and research institutions in France or abroad, or from public or private research centers.
L'archive ouverte pluridisciplinaire HAL, est destinée au dépôt et à la diffusion de documents scientifiques de niveau recherche, publiés ou non, émanant des établissements d'enseignement et de recherche français ou étrangers, des laboratoires publics ou privés. 


\title{
Towards a better understanding of wood cell wall characterisation with contact resonance atomic force microscopy
}

\author{
O. Arnould ${ }^{\mathrm{a}, *}$, R. Arinero ${ }^{\mathrm{b}}$ \\ a Laboratoire de Mécanique et Génie Civil (LMGC), Université de Montpellier, CNRS, Montpellier, France \\ ${ }^{\mathrm{b}}$ Institut d'Electronique et des Systèmes (IES), Université de Montpellier, CNRS, Montpellier, France
}

\begin{abstract}
A B S T R A C T
Keywords:

A. Wood

B. Mechanical properties

C. Fibres

D. Mechanical testing

Nowadays, the multi-scale modelling of wood has a great need for measurements of structural, chemical and mechanical properties at the lowest level. In this paper, the viscoelastic properties in the layers of a wood cell wall are investigated using the contact resonance mode of an atomic force microscope (CR-AFM). A detailed experimental protocol suitable for obtaining reproducible and quantifiable data is proposed. It is based on three main steps: sample preparation to obtain a good surface state, calibration of the contact modulus using reference samples, and image processing to produce the viscoelastic images. This protocol is applied on chestnut tension wood. The obtained topography and semi-quantitative viscoelastic maps are discussed with respect to the cell wall structure, sample preparation effects, and AFM measurement specificity compared with nanoindentation.
\end{abstract}

\section{Introduction}

Wood is produced in successive cylindrical layers during the radial secondary growth of trees by a very thin layer of living cells located under the bark and called cambium. It is composed of several kinds of cells, organised into a honeycomb-like structure, that have a particular role for the tree [1]. From a mechanical point of view, fibres, or tracheids, are the cells that compose the tree's skeletal structure. Fig. 1a shows the typical multi-layered structure of a normal wood fibre. Each wood cell wall layer plays a particular role in the mechanical behaviour of wood, and the macroscopic longitudinal elastic properties of normal wood originate mainly in those of its secondary cell wall layer, $S_{2}$ (Fig. 1a). All layers of the secondary wall can be seen as a unidirectional long fibre composite whose fibres are crystalline (at around 70\%) cellulosic microfibrils (a priori as long as the cell and with some nanometres in diameter), and the matrix is made up of amorphous polymers: hemicelluloses, lignin and extractives [3]. The microfibrils are helicoidally oriented in each layer of the secondary wall [4]; for example, they are inclined to the cell axis by the so-called microfibril angle (MFA), usually devoted to the angle in the $S_{2}$ layer. Moreover, fibres act like a muscle for the tree axis [5] and their (ultra)structure can differ from that of a classical structure (i.e. normal wood) in the case of strong axis reorientation where reaction

\footnotetext{
* Corresponding author. Tel.: +33 (0)4 671496 50; fax: +33 (0)4 67144772 . E-mail address: olivier.arnould@univ-montp2.fr (O. Arnould).
}

wood is produced [6,7]. Hardwood trees, for example, produce the so-called tension wood fibres that may have a supplementary layer, generally in addition to and within the $S_{2}$ layer, as shown in Fig. $1 \mathrm{~b}$, with an MFA close to $0^{\circ}[6,7]$. This layer is usually called the "G layer" because its matrix has a gel-like structure $[6,8]$. This study is part of a research project on hardwood tree biomechanics that is focused particularly on the development and evolution of the mechanical properties of the G layer.

The variability in wood cell distribution, thickness and properties makes it difficult to study the mechanics of wood at the macroscopic scale (e.g. tree rings). Indeed, every tree and species has its own cellular organisation and structure, both of which have a strong effect on the behaviour at the macro-scale. Thus, multi-scale modelling of wood, which is used to predict, for example, the longterm behaviour of wood in structural applications, is very much dependent on the measurement of wood properties at the lowest level $[9,10]$.

The mechanical properties at the cell wall level can be estimated using numerical computations of the properties of its constituents or, as is more usual and traditionally done, the estimations can be carried out experimentally on chemical compounds extracted from the cell walls [3]. Other measurements consist of "classical" tensile tests at the scale of the tree rings or tissue using back-calculations [11] and/or specific strain field measurements $[12,13]$. See Gamstedt et al. [14] for an extensive review on the use of mixed experimental-numerical methods to characterise wood properties that are not easily available by direct 


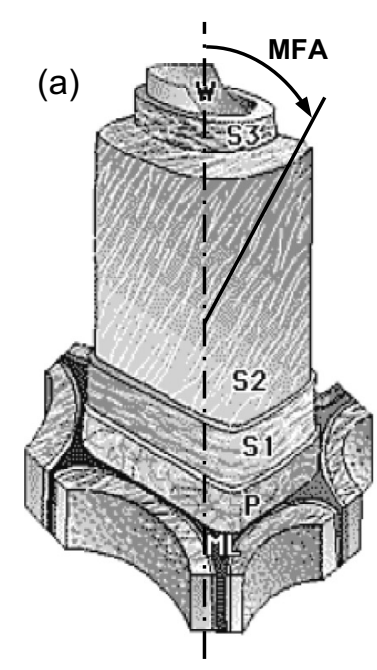

(b)

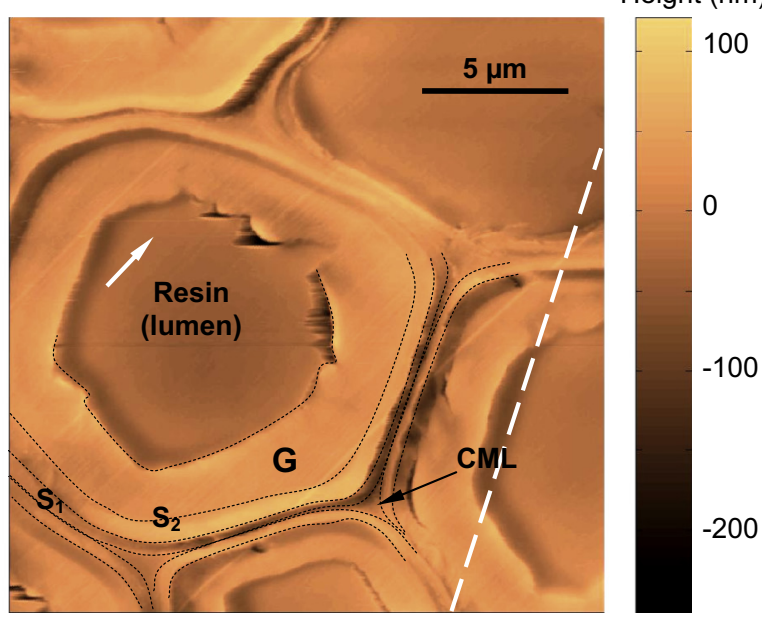

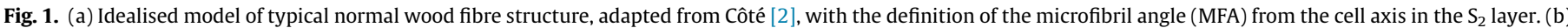

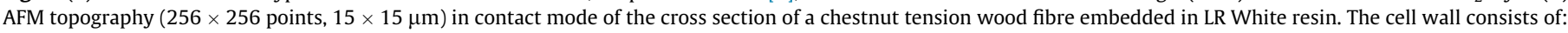

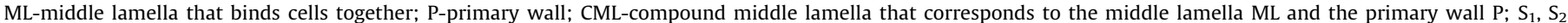

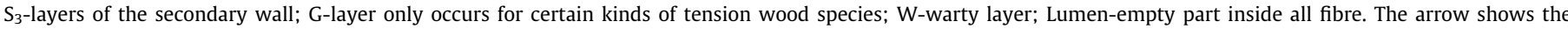

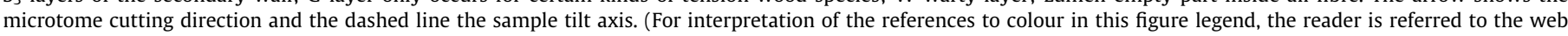
version of this article.)

measurements. Direct measurement at the whole cell wall scale is possible using single fibre testing and a new technique based on focused ion beam (FIB) machining of the cell wall [15]. Nanoindentation is one of the most promising and frequently used techniques nowadays. It provides access to the in situ mechanical properties within cell walls with as few modifications as possible; nevertheless, it does not provide access to the elastic properties but only to a complex combination of them through the so-called indentation modulus [16]. This technique has already been applied to estimate the indentation modulus of some of the wall layers of native or thermo-mechanically modified cells [15]. However, as it is commonly accepted that the radius of the elastically affected volume around the indenter is about three times the residual indent size for an isotropic material [17], this technique requires the layer thickness to be at least three times greater than the indent size; that is, typically on the order of a micrometre, to avoid measurement artefacts [18]. As the width of the cell wall layers varies from about $0.1 \mu \mathrm{m}$ (primary wall) to less than $10 \mu \mathrm{m}\left(\mathrm{S}_{2}\right.$ and/or G layer), the interpretation of the measurements obtained by nanoindentation in the presence of a properties gradient or within a thin layer is not straightforward due to boundary effects.

Atomic force microscopy (AFM) allows not only topographic mapping at the nanometre scale but also the measurement of some of the mechanical properties (elasticity, viscosity, etc.) at this scale. Mechanical measurements by AFM, using force-distance curves, force modulation microscopy, and so on, require approaches similar to those in nanoindentation but with a spatial resolution on the order of some tens of nanometres [19]. In our case, we use contact resonance AFM (CR-AFM) mode, which is a part of the more general atomic force acoustic microscopy (AFAM) [20,21] that has already been applied on wood [22-24]. These latter studies mainly focused on the feasibility of this technique and the disclosure of the first results. While CR-AFM has now reached a certain level of maturity, the present article aims to describe a complete experimental protocol, from sample preparation to image processing. It also intends to provide a better interpretation of the images obtained on the wood cell wall. The study is restricted to the case of the chestnut tension wood cell and demonstrates local semiquantitative measurements and qualitative mapping of the viscoelastic properties at the ultrastructural level in wood science.
Furthermore, the specific structure of tension wood fibres allows us to highlight the sample preparation artefacts and to discuss them, as well as the interpretation of the CR-AFM images.

\section{Materials and methods}

\subsection{Material and sample preparation}

In order to investigate the CR-AFM imaging technique in the case of wood, chestnut (Castanea sativa Mill.) tension wood was selected for its ability to produce a thick G layer. This allows easier measurements in CR-AFM (i.e. high surface area with reduced topography) and mechanical contrast is more likely to be observed. All mechanical measurement based on indentation requires samples with a surface as flat as possible compared to the contact radius in order to be able to accurately estimate the contact area. Moreover, in the case of AFM, the tip is very brittle and surface roughness must be as low as possible to obtain reliable mechanical measurements and reduce breakage risks.

The wood samples are then embedded in a resin in order to fill the lumen and decrease the surface roughness by reducing deformation during the cutting process. Sticks $(1 \mathrm{~cm}$ in longitudinal direction, $1 \times 1 \mathrm{~mm}^{2}$ in transverse section) are trimmed off by splitting to guarantee a good axial direction. They are then cut manually with a razor blade to produce a clear transverse surface and obtain cubes of about $1 \mathrm{~mm}^{3}$. The samples are dehydrated with ethanol series (50\%, 75\%, 90\% and 100\%) under vacuum and embedded in an increasing ratio of LR White acrylic resin [25] using an ordinary gelatine capsule as mould. Dehydration is necessary for proper embedding and curing of the LR White resin, which is hydrophobic. Note that, in the present case, the sample could not be dried out before embedding, to avoid the dehydration with ethanol, as it would have damaged the gelatinous matrix of the $G$ layer. Moreover, it has been shown that ethanol is a solvent with a low impact on the cell wall state [26]. LR White resin is dedicated to biological samples and is assumed to behave like epoxy embedding resins, such as Spurr or Agar, which have reduced penetration into the wall of normal undamaged wood [27,28]; to our knowledge, however, no studies have investigated the case of the G layer. Resin penetration into samples, especially into this layer, should be 
checked in future studies by, for example, scanning thermal microscopy [28] or a spectroscopic technique like the Raman technique [29]. The use of an embedding medium is necessary here to maintain the cell wall structure during the next step of the sample surface preparation, particularly to prevent G layer detachment [30]. Note that this can be avoided for a normal wood sample [31], but it requires precaution to prevent AFM tip breakage during the experiments.

After resin polymerisation in an oven $\left(60^{\circ} \mathrm{C}\right.$ overnight), the whole resin block with the sample is machined to reduce its cross section. A rotary microtome (Leica RM2265) is then used first with a glass knife to remove the first $100 \mu \mathrm{m}$ of material containing the border effects that occur during the initial sample preparation [30]. Then a diamond knife (Diatom Histo) is used to cut a series of very thin sections (about $500 \mathrm{~nm}$ thick) at reduced cutting speed to minimise compression and sample deformation during the cutting process. The resulting topography of the remaining wood block sample surface is obtained simultaneously during the mechanical measurement in CR-AFM mode (Fig. 1b). Some steps in the topography are observed due to differences in the stiffness or the cutting behaviour of the different layers. However, the typical root-mean-square (RMS) roughness in the thicker layers, $G$ and $\mathrm{S}_{2}$, is usually less than $10 \mathrm{~nm}$.

\subsection{CR-AFM measurements}

In the present study, the CR-AFM setup consists in applying a small periodic force to the AFM cantilever by means of an electrostatic potential between the reflective coating of the cantilever and the sample holder. This mode of excitation allows us to reduce measurement noise and artefacts, and this was possible in our case as the sample is not conductive [32,33]. When the excitation frequency is close to a natural frequency of the cantilever in contact with the sample surface through its tip, a cantilever resonance occurs. Basically, the measurement is then based on performing a frequency sweep at a given point on the surface and recording the cantilever vibrations through the AFM photodetector. According to several authors [20,21,34], the resonance frequency, $f_{0}$, and the quality factor, $Q$, of the obtained frequency spectrum can be related to the real, $k^{\prime}$, and imaginary, $k^{\prime \prime}$, parts of the contact stiffness at the resonance frequency. Note that in our case we only use the first resonance frequency of the cantilever.

It is then necessary to use the appropriate contact model to derive the contact modulus $M$ of the tested volume of material from the contact stiffness $[17,21,35,36]$. We assume that the contact stiffness is mainly due to the linear viscoelastic response of the material and we neglect the contribution of the lateral contact stiffness due to the AFM cantilever tilt (typically $12^{\circ}$ here), which leads to a loading that is not purely normal to the surface [32]. Moreover, the studied materials are such that the so-called DMT model [35] can approximate the contact stiffness, and particularly its real part that is linked to $f_{0}[34]$ :

$k^{\prime}=\left(6 M^{\prime 2} R\left(F+F_{\mathrm{a}}\right)\right)^{1 / 3}$,

where $R$ is the tip apex radius, $F$ the applied mean force, $F_{\mathrm{a}}$ the adhesion force and $M^{\prime}$ the real part of the viscoelastic contact modulus at the resonance frequency. In the present case, the studied materials are not highly viscous (see the value of the loss tangent below) and we assume that $M^{\prime}$ is close to the quasi-static elastic contact modulus $M$. If the tip is much stiffer than the sample, as is the case here, and the sample elastic isotropic, $M=E /\left(1-v^{2}\right)$ where $E$ and $v$ are, respectively, its elastic modulus and Poisson's ratio [17]. The loss tangent, $\tan \delta=k^{\prime \prime} \mid k^{\prime}$, characterises the viscous dissipation of the material in the tested volume. For low values (i.e. more or less lower than 0.01 , see below), it corresponds to the reciprocal of the quality factor $Q$ of the cantilever if all other dissipation processes like, for example, tip sliding friction, viscosity due to capillary force, or fluid damping of the cantilever, are neglected $[37,38]$. The last step should be to identify the independent material viscoelastic parameter(s). This is not so obvious in the case of an anisotropic and fibrous material such as wood cell wall layers [39], even though an anisotropic indentation model was applied to a typical $S_{2}$ layer [16] with reverse identification of some of the elastic properties [40]. For this reason, this issue is not addressed in this paper. Last, without any significant modification in the AFM device, this method theoretically offers submicrometre spatial resolution, as the mean and vibrating applied forces can be tuned as low as possible, and this allows significantly reduced lateral force effects [41]. Moreover, by scanning the surface at a constant mean force and excitation frequency $f_{\text {im }}$, reasonably fast mapping (typically $\sim 45 \mathrm{~min}$ per $256 \times 512$ image for $0.1 \mathrm{~Hz}$ slow scan axis frequency, see below) of the resonance frequency and quality factor is possible whilst acquiring the sample topography, without doing a frequency sweep at each point. This imaging technique is based on the cantilever vibration parameters (i.e. the real and imaginary parts) that are directly linked to the parameters of the resonant spectrum modelled as a Lorentzian [34]. This mathematical processing requires $f_{\text {im }}$ to be close to $f_{0}$; that is, $f_{\text {im }}$ must be choice in the resonance peak range, especially for the back-calculation of $Q$.

In our case, a commercial AFM (Veeco Enviroscope) is used and the photodetector signal is analysed by a lock-in amplifier (EG\&G model 5302) to extract the real and imaginary parts of the cantilever vibration generated by the electrostatic excitation through an external generator (FLUKE PM5138A). In order to achieve the frequency sweep and resonance spectra acquisitions during the calibration, an automated measurement is realised using Labview software and the GPIB National Instruments interface. The (average) technical specifications of the probe used for the experiments (Nanoworld Arrow FMR) are: bulk silicon; cantilever: thickness $3 \mu \mathrm{m}$, length $240 \mu \mathrm{m}$, width $35 \mu \mathrm{m}$, stiffness $2.8 \mathrm{~N} / \mathrm{m}$, free natural frequency $75 \mathrm{kHz}$ and aluminium conductive and reflective coating on the back; tip: average radius estimated to be around $55 \mathrm{~nm}$ during the measurements, using calibration grating (NT-MDT TGT-01) [42]. This grating allows for a kind of (fast) reverse imaging of the actual apex shape, with enough accuracy in the present case. The total mean applied force is estimated to be around $180 \mathrm{nN}$ and the sinusoidal electrostatic excitation is applied with an amplitude of $10 \mathrm{~V}$.

Our experiments show that the use of a theoretical model of cantilever vibration for the back-calculation of the contact modulus is not so obvious. We thus decided here to use a series of "reference" materials [36] like more or less stiff polymers (Polyurethane PU, Polyester PE, PMMA) and stiffer and less viscous materials like monocrystalline sulphur or glass. Frequency spectra are done on the reference samples at several fixed points with the same mean applied force, including the adhesion force, and electrostatic excitation, as during the experiments on wood. The obtained resonance frequencies are then compared to the contact moduli, estimated using Hertz theory [17] for isotropic media (like polymers and glass) or its extended version from Delafargue and Ulm [43] for anisotropic media (like monocrystalline sulphur), to build a master curve. It is worth to mention that theses samples were characterised by a combination of nanoindentation and ultrasound measurements (at $1 \mathrm{MHz}$, close to the frequency range of the CR-AFM measurements). The contact modulus of unknown samples (wood cell wall here) is extrapolated from this curve. The strongest assumptions here are that: (i) these reference samples are homogeneous, even at the submicrometre scale, and their properties at this scale are comparable to those at the macroscopic (ultrasound) scale [44-46]; (ii) the tip shape apex and the total applied force, including adhesion, are constant all along the measurements (see Eq. (1)); and (iii) the resonance frequency of 
the cantilever is the same in fixed point position and scanning mode [47]. For this last issue and for cantilever vibration stability, the frequency of the fast scan axis is set as low as possible: $0.1 \mathrm{~Hz}$. Moreover, the shape of the AFM cantilever tip apex (i.e. mean radii of curvature) is checked during calibration and the experiments on wood as wear can occur, especially for the first measurements, and lead to a drastic change in the tip apex shape $[21,36,45,48]$. A fast check during measurement is possible by measuring the resonance frequency on the embedding resin, used as a reference material. The real tip shape is measured using the aforementioned calibration grating before and after all measurements. Last, the adhesion force is measured at many points on the whole sample by doing a force-distance curve and is quite constant at around $40 \mathrm{nN}$. Note that all measurements are made in ambient environmental conditions without any fine local control of the temperature or relative humidity.

\section{Results and discussion}

\subsection{Sample topography}

The diagonal straight lines in Fig. 1b result from imperfections in the edge of the diamond knife and indicate the cutting direction (see the arrow, also). It is interesting to note that height varies in the same manner (high and low diametrically opposite) around the axis of all the cells for the $S_{1}$ and $S_{2}$ layers, whereas it remains almost constant for the compound middle lamella (CML), the $G$ layer and the embedding resin in the lumen. These observed height variations can be mainly explained by the different orthogonal cutting behaviours of the layers due to their respective MFAs, and particularly the orientation of the microfibrils to the cutting direction [49]: A layer whose microfibrils are orthogonal to the cutting direction might be more resistant than one with microfibrils parallel to the cutting direction. Moreover, the component of the force exerted by the knife normal to the surface of the section induces out-of-plane deformations, which will depend on the mechanical behaviour of the layer linked again to the MFA, that generate variations in surface topography. A similar behaviour occurs in the orthogonal cutting of, for example, multidirectional carbon/epoxy composites. Iliescu et al. [50] studied the effect of the fibre orientation to the cutting direction in this kind of composite. They showed that surface quality is worse and height is lower when the fibre direction is opposed to the cutting direction. This can be confirmed here by comparing the surface topography of Fig. 1b with the simulated theoretical map (using Matlab software) of the angle $\alpha$ in Fig. 2 for a typical cell wall. This angle corresponds to the apparent MFA projected in the plane containing the cutting direction and the normal to the surface. $\alpha$ is set positive if the microfibril direction is in the cutting direction, and negative otherwise: the greater the angle, the higher the surface and the better its quality. $\alpha$ is constant for the G layer, as its MFA is close to zero [6], and so is the surface quality and height. CML and resin areas are made of almost amorphous polymers and are not, or are less, affected by this "cutting" effect. Finally, the orientation of the helix made by the microfibrils in the $S_{1}$ and $S_{2}$ layers is opposite; that is, the microfibrils in the $S_{1}$ layer are most probably mainly arranged in the so-called S-helix (left-hand thread helix, MFA $>90^{\circ}$, see Fig. 1a), whereas they are arranged in the so-called Z-helix (right-hand thread helix, MFA $<90^{\circ}$, see Fig. 1a) in the $S_{2}$ layer [4,51]. This explains the opposite trends in these two layers in Fig. 1b. Note that the $S_{1}$ layer has not been represented in Fig. 2, for the sake of clarity, as $\alpha$ may vary from almost $-90^{\circ}$ to $90^{\circ}$.

\subsection{CR-AFM spectra and contact modulus}

The CR-AFM experiments are performed in two steps. The first step is to engage the tip in contact with the sample and to carry out frequency spectra at different points at almost the same position in each layer. In Fig. 3, the amplitude of the output signal of the photodetector is plotted as a function of the electrostatic excitation frequency. The first resonance frequency $f_{0}$ and the quality factor $Q$ of the frequency spectrum in each layer are then deduced. The contact modulus in the longitudinal direction $M_{\mathrm{L}}$ is then computed using the master curve obtained on the reference materials (as depicted in the previous section). The results are summarised in Table 1. They correspond to the mean values obtained from at least three spectra done in three different but close positions within the same layer. The uncertainty in this table only represents the variation in the value computed from one point to another. It does not take into account all the uncertainty coming from the different calibration steps or the measurement accuracy, which may be much higher. These results are consistent with those obtained previously on oak [22]. They are in the same order of magnitude, but lower, than what is usually obtained by nanoindentation on other kinds of wood species; for example, a $0^{\circ}$ MFA yields an indentation modulus around $14-20 \mathrm{GPa}$ in nanoindentation $[15,16,40]$. This is all the more true if we consider the viscous nature of the cell wall layers and the huge difference in the frequency measurements between nanoindentation and CR-AFM. The lower values obtained here may be partly explained by the anisotropic and fibrous nature of the material, which could lead to differences in contact behaviour between nanoindentation

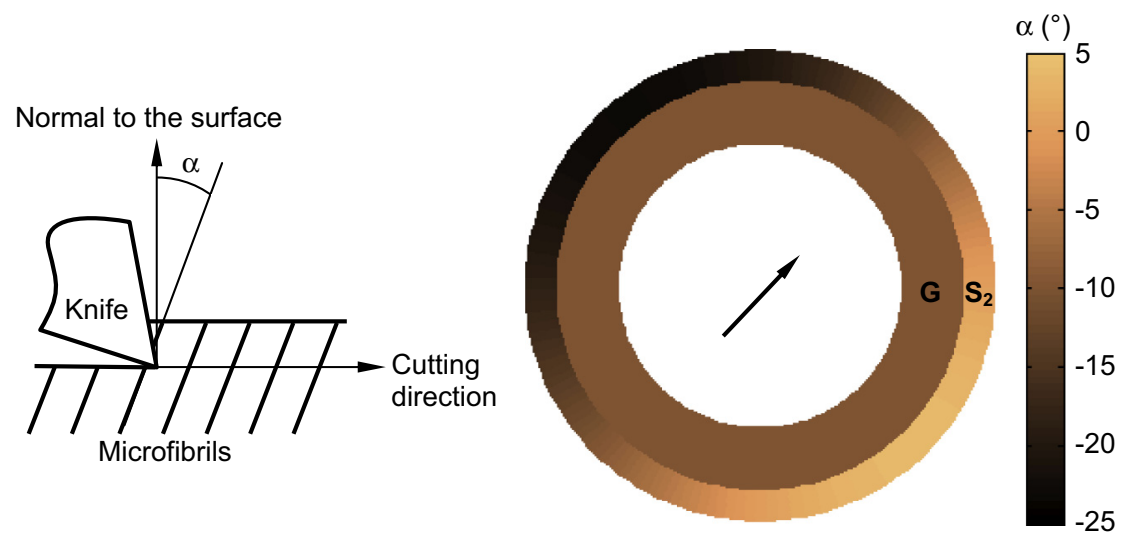

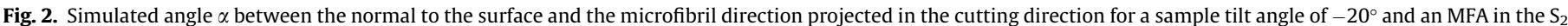

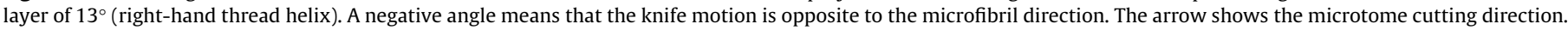
(For interpretation of the references to colour in this figure legend, the reader is referred to the web version of this article.) 


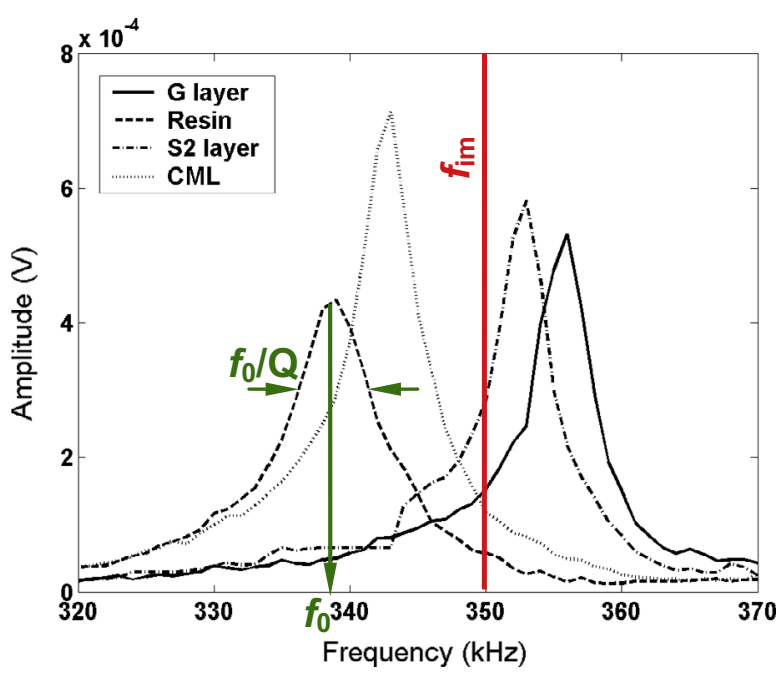

Fig. 3. Amplitude frequency spectrum for the different layers. Definition of the resonance frequency $f_{0}$ and quality factor $Q$ in the case of the embedding resin. Choice of the imaging frequency $f_{\text {im }}$ for Fig. 4 . (For interpretation of the references to colour in this figure legend, the reader is referred to the web version of this article.)

("deep" penetration depth of some hundreds of nanometres using a diamond Berkovich 3-sided pyramid indenter) and the present measurements ("small" penetration depth of some nanometres with an almost spherical tip apex). This effect could be enhanced by the cantilever tilt in CR-AFM, which yields a "non-normal" loading of the surface, contrary to nanoindentation, and makes the measurement more or less sensitive to the transverse contact stiffness, which is lower than the normal one here due to the anisotropy of the cell wall. Moreover, the cutting process inevitably leads to near surface modification with probably a significant impact on the viscoelastic properties (e.g. creating nanocracking or molecular reorientation in the wake of the knife). CR-AFM measurements at lower scales, closer to the microfibril scale, combined with other sample surface preparations could help to evaluate these modifications. Surface preparation could also be improved by using an instrumented microtome [52] and/or by comparing it with other kinds of sample surface preparation [53]. Last, the fibrous structure of the cell wall leads to nanometre topography linked to the cellulose aggregates [49,53]. This nano-roughness can lead to an overestimation of the real contact area [36] and yields an estimated contact modulus lower than the real one for a given contact stiffness. CR-AFM, as a near surface measurement, is far more sensitive to these near surface modification and nanoroughness effects than nanoindentation.

\subsection{Viscoelastic mapping}

The second step of the measurement is mapping the surface at a given cantilever excitation frequency, $f_{\text {im. }}$. To be able to compute the resonance frequency and quality factor from the real and imaginary parts of the cantilever vibration, $f_{\text {im }}$ must be chosen as

Table 1

Contact modulus $M_{\mathrm{L}}$ and reciprocal of the quality factor $Q^{-1}(\sim \tan \delta$ at the resonance frequency) in the longitudinal direction for the different layers of the cell wall computed from the cantilever resonance spectra. Specified uncertainties correspond to the variations between 3 measurement locations.

\begin{tabular}{lll}
\hline & $M_{\mathrm{L}}(\mathrm{GPa})$ & $Q^{-1}$ \\
\hline Resin & $3.5 \pm 1.5$ & $0.013 \pm 0.002$ \\
CML & $6 \pm 0.5$ & $0.009 \pm 0.003$ \\
$\mathrm{~S}_{2}$ & $13 \pm 0.5$ & $0.006 \pm 0.003$ \\
$\mathrm{G}$ & $15 \pm 1.5$ & $0.009 \pm 0.003$ \\
\hline
\end{tabular}

close as possible to the resonance frequency on each layer. In the present case, as we want to demonstrate the ability of this technique to sense differences in mechanical properties mainly in the $S_{2}$ and $G$ layers, the imaging frequency is chosen close to that obtained on these layers (Fig. 3). As a result, the mechanical properties of the embedding resin and, to a lesser extent, that of the CML will be less well estimated, especially concerning the quality factor $Q$. The resulting contact modulus and reverse of the quality factor maps are given in Fig. 4 for an imaging frequency $f_{\text {im }}=350 \mathrm{kHz}$.

In Fig. 4a, the $S_{2}$ and $G$ layers clearly appear to be the stiffest ones on average, whereas the $S_{1}$ and CML layers are the softest on average, as in Table 1 . The layers appear far more clearly than in the topographic image of Fig. 1b. Opposite variations in the contact modulus of the $S_{1}$ and $S_{2}$ layers of all the cells are observed, whereas the modulus is almost constant in the $G$ layer. For the $\mathrm{S}_{2}$ layer, the contact modulus variations are from $\sim 8$ to $\sim 13 \mathrm{GPa}$, while it is around $11 \mathrm{GPa}$ on average for the G layer. First, these values are slightly lower than those obtained in the first measurement step by doing the frequency spectra at fixed points. This is most probably due to the differences in the contact behaviour between a fixed and a sliding tip [47]. Further development is (a)
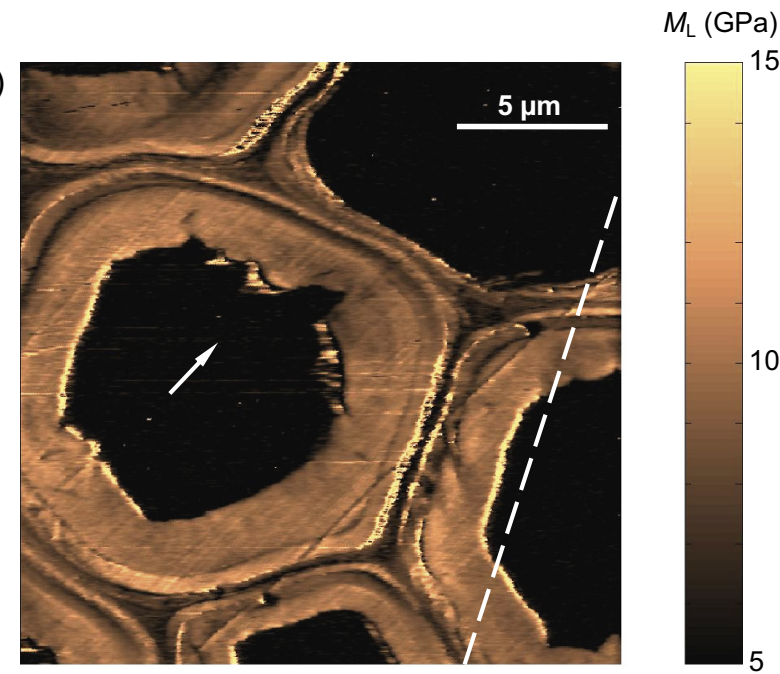

(b)

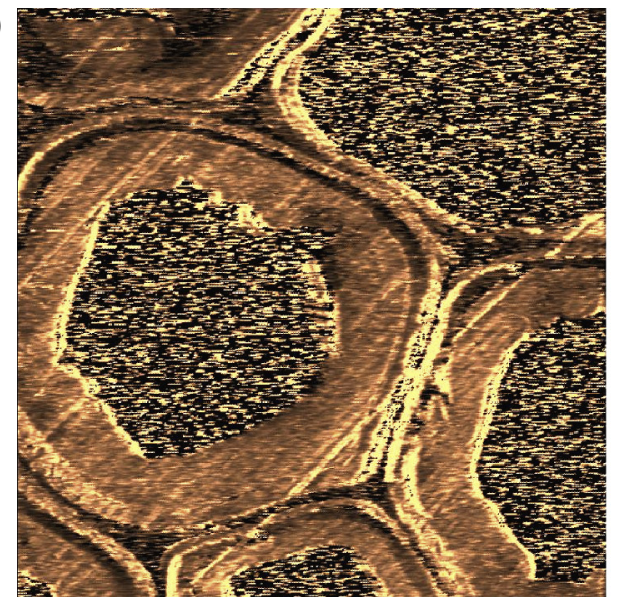

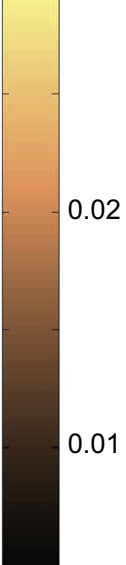

Fig. 4. Semi-quantitative mapping at $350 \mathrm{kHz}$ in the same area as in Fig. 1: (a) longitudinal contact modulus $M_{\mathrm{L}}$. The arrow shows the microtome cutting direction and the dashed line the sample tilt axis; (b) reciprocal of the quality factor $Q^{-1}$ ( $\sim \tan \delta$ at the resonance frequency). (For interpretation of the references to colour in this figure legend, the reader is referred to the web version of this article.) 
needed to be able to do the calibration on the reference materials in scanning mode. Second, comparing Figs. $1 \mathrm{~b}$ and $4 \mathrm{a}$ shows that cutting effects, highlighted by the topography and discussed in the previous section, are almost not correlated with the evolution of the contact modulus in the $S_{1}$ and $S_{2}$ layers. At first glance, we might thus assume that the cutting effects on the contact modulus are almost the same, whatever the apparent MFA. These contact modulus variations are therefore most probably due to an oblique cross section combined with the MFA of each layer. It is indeed difficult to control the sample orientation, and even more so for the local fibre orientation, as the embedding capsules are opaque. This induces a different type of loading with respect to the microfibril axis; that is, the apparent MFA varies around the cell axis [40,54]: the highest modulus would then mean a lower apparent MFA. The fibre tilt axis can reasonably be assumed to be parallel to the line that goes through the extreme values of the contact moduli in the $S_{1}$ and $S_{2}$ layers (see the dashed line in Figs. $1 \mathrm{a}$ and $4 \mathrm{a}$ ). The fact that the contact modulus is at its maximum (respectively, minimum) in the $S_{1}$ layer while it is at its minimum (respectively, maximum) in the $S_{2}$ layer could be explained again by the opposed $S$ - and Z-helixes in the $S_{1}$ and $S_{2}$ layers, respectively (see the previous section dealing with the sample topography). Furthermore, the contact modulus is almost constant in the G layer, as the MFA is close to zero in this layer. It is possible to theoretically compute the effect of such a tilt on the contact

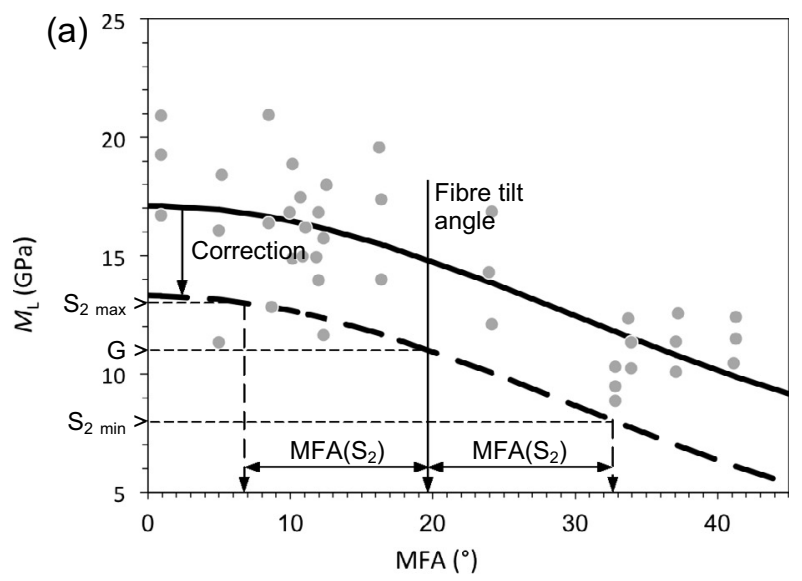

(b)

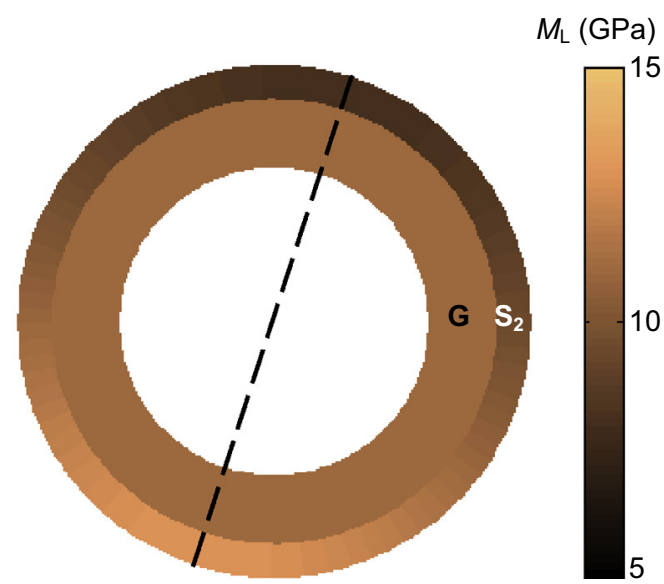

Fig. 5. (a) Nanoindentation data (light grey dots) and mean theoretical curve (solid line) of the contact modulus versus the MFA from [40]; corrected theoretical curve for the present AFM measurements (dashed line) (b) simulated contact modulus for a sample tilt angle of $-20^{\circ}$ and MFA in the $S_{2}$ layer of $13^{\circ}$ using the corrected theoretical curve. The dashed line is parallel to the sample tilt axis. (For interpretation of the references to colour in this figure legend, the reader is referred to the web version of this article.) modulus. For example, one can use the theoretical curve of the evolution of the indentation modulus versus MFA, $M_{\mathrm{L}}(\mathrm{MFA})$, from Jäger et al. [40], for a typical $S_{2}$ layer, as shown in Fig. 5a. This curve has been fitted on nanoindentation measurements and must then be corrected here to take the aforementioned effects - that is, of cutting and the specificity of the AFM on the measured mechanical properties - into account. We suggest to simply translate this curve down and assume that it is valid for the $S_{2}$ as well as the $G$ layer; that is, whereas it is known they are not similar in chemical composition [6,7]. As depicted in Fig. 5a, the apparent MFA corresponding to the mean contact modulus in the $\mathrm{G}$ layer is theoretically equal to the fibre tilt angle. Moreover, the apparent MFAs for the extreme values in the $S_{2}$ layer correspond to this tilt angle plus or minus the real MFA in the $S_{2}$ layer. So the translation is done such that the measured moduli correspond to apparent MFAs that fulfil the aforementioned requirement, as depicted in Fig. 5a. An order of magnitude of the real MFA in the $S_{2}$ layer, close to $13^{\circ}$, and of the fibre tilt angle, close to $-20^{\circ}$, are obtained. Whereas the value of the MFA is plausible, that for the tilt angle seems a bit too large, but it has to be set back in the light of all the assumptions made here. A more reliable value of these angles will require measurements using polarised light or Raman spectroscopy $[40,54,55]$. To finally confirm the effect of the fibre tilt, we use these angles to simulate the apparent MFA for a perfect cell wall and the corrected $M_{\mathrm{L}}$ (MFA) curve to compute the evolution of the contact modulus in these theoretical $S_{2}$ and G layers (Fig. 5b). Comparison of the measurements in Figs. $4 \mathrm{a}$ and $5 \mathrm{~b}$ shows quite good agreement in spite of all the assumptions made. Thus, this strengthens the assumption of a fibre tilt associated with a near surface effect and the measurement specificities in AFM to explain the evolution of the contact modulus within the cell wall layers.

Last, it can be seen that topography (e.g. scratches due to cutting and step height to a lesser extent, see Fig. 1b) has an effect on measurement [24], especially for thin layers like $S_{1}$ where the variation in the topography is high. Whatever the real origin of all these observations, this demonstrates the ability of CR-AFM to highlight elastic property variations within a single cell wall layer.

For the viscous parameter, $\tan \delta$ (assumed to correspond to the reciprocal of the quality factor $Q$ ) in Fig. $4 \mathrm{~b}$, the results are not so clear as the CML seems to be surprisingly less viscous than the $S_{2}$ layer, whereas the $S_{1}$ is more viscous. This is in contradiction with values of $Q^{-1}$ obtained in Table 1 and it seems to be mostly due to the choice in the imaging frequency, which is too far from the resonance frequency of the CML (see the noise in the resin part, also). It is interesting to note that, on average, the $G$ layer is more viscous than $\mathrm{S}_{2}$, as in Table 1 . This could be due to the gel-like structure of the matrix in this layer. Again, the effect of the fibrous nature of the layers on the contact properties is questioned, as is the resulting cutting effect on nano-roughness and other probable structural near surface modifications. Note that the topography has a stronger effect in this case. More work is needed to address this aspect of the measurements, especially work on how to compute and correct the $Q$ factor from other, here neglected, dissipative mechanisms $[37,38]$. Last, one can wonder about the physical meaning (in the frame of tree biomechanics or the usual use of wood as a material) of viscosity measured at such a high frequency.

\section{Conclusions}

In this paper, we have shown that CR-AFM applied to the wood cell wall, or any kind of natural fibre cell wall, allows semiquantitative estimation of the contact modulus for a given (fixed) point or in scanning mode with high spatial lateral resolution. Both calibration with a set of reference samples and cantilever signal processing are used to determine the contact modulus. 
Because such AFM-based experiments are strongly influenced by the surface topography of the samples, a detailed preparation procedure is proposed, based, inter alia, on the impregnation of a resin and a cutting protocol. The study is conducted on the chestnut tension wood cell as its typical microstructure, especially with a thick G layer, allows us to highlight sample preparation artefacts due to cutting effects and wood fibre misalignment. The obtained contact moduli are, on average, consistent with but lower than the values from the literature obtained by nanoindentation. The findings are interpreted by notably taking into account, at this state of our investigations, the possible artefacts related to sample preparation, particularly near surface modifications due to cutting, and the specificity of AFM measurements compared with nanoindentation. Similar effects and trends of the sample tilt on the contact moduli have been observed for CR-AFM and nanoindentation.

Works are in progress to improve the calibration and experimental conditions to make the estimation of the viscoelastic properties more quantitative by using a softer cantilever coupled with higher harmonics $[21,56]$ and a dual-resonance frequency tracking setup [57,58]. This will improve the sensitivity of the measurements, with improved lateral spatial resolution, and will yield more reliable results in all layers, even in the case of high mechanical contrasts. A future goal is to continue to go down the scale and try to obtain an estimation of the in situ mechanical properties of the microfibrils and surrounding matrix. Another AFM technique, ultrasonic force microscopy [59], was recently used on similar samples and gave encouraging preliminary results at the microfibril scale. Moreover, CR-AFM measurements could be coupled with another kind of cantilever excitation to measure other "averaged" elastic properties of the anisotropic cell wall layer [60,61] and/or be combined with an intentional - that is, controlled - tilt sample and measurements of the local apparent MFA [54,55] to extract components of the cell wall layer elastic tensor [40]. Concerning the estimated damping properties of the cell wall layers, improvement in the data analysis will be required to take other dissipative mechanisms into account $[37,38]$ and to understand the values obtained at such high frequencies. Finally, the effect of the environmental conditions, and especially the real moisture content of each cell wall layer, must be evaluated.

\section{Acknowledgements}

The authors acknowledge K. Bytebier (LMGC, Université de Montpellier, France) and J.Y. Ferrandis (IES, Université de Montpellier, France) for their participation and help in the measurements and D. Laux (IES, Université de Montpellier, France) and Andreas Jäger (TU Wien, Austria) for the calibrations of the reference polymer materials using ultrasound and nanoindentation, respectively. Financial support by the CNRS (NanoMecPar project), the Université de Montpellier and the COST Action FP0802 is gratefully acknowledged. Part of this work was performed in the framework of the projects "Analogs" (ANR-BLAN08-3_310735) and "StressInTrees" (ANR-12-BS09-0004) funded by the French National Research Agency (ANR).

\section{References}

[1] Thibaut B, Gril J, Fournier M. Mechanics of wood and trees: some new highlights for an old story. CR Acad Sci IIb 2001;329:701-16.

[2] Côté WA. Wood ultrastructure - an atlas of electron micrography. Seattle: University of Washington Press; 1967.

[3] Salmén L. Micromechanical understanding of the cell-wall structure. CR Biol 2004;327:873-80.

[4] Meylan BA, Butterfield BG. Helical orientation of the microfibrils in tracheids, fibres and vessels. Wood Sci Technol 1978;12:219-22.
[5] Fournier M, Alméras T, Clair B, Gril J. Biomechanical action and biological functions. In: Gardiner B, Barnett J, Saranpää P, Gril J, editors. The biology of reaction wood. Berlin: Springer-Verlag; 2014. p. 139-69.

[6] Ruelle J. Morphology, anatomy and ultrastructure of reaction wood. In: Gardiner B, Barnett J, Saranpää P, Gril J, editors. The biology of reaction wood. Berlin: Springer-Verlag; 2014. p. 13-36.

[7] Fagerstedt KV, Mellerowicz E, Gorshkova T, Ruel K, Joseleau JP. Cell wall polymers in reaction wood. In: Gardiner B, Barnett J, Saranpää P, Gril J, editors. The biology of reaction wood. Berlin: Springer-Verlag; 2014. p. 37-106.

[8] Clair B, Gril J, Di Renzo F, Yamamoto H, Quignard F. Characterization of a gel in the cell wall to elucidate the paradoxical shrinkage of tension wood. Biomacromolecules 2008;9:494-8.

[9] Hofstetter K, Gamstedt EK. Hierarchical modelling of microstructural effects on mechanical properties of wood. A review. Holzforschung 2009;63:130-8.

[10] Salmén L, Burgert I. Cell wall features with regard to mechanical performance. A review. Holzforschung 2009;63:121-9.

[11] Cave ID, Hutt L. The longitudinal Young's modulus of pinus radiata. Wood Sci Technol 1969;3:40-8.

[12] Forsberg F, Mooser R, Arnold M, Hack E, Wyss P. 3D micro-scale deformations of wood in bending: synchrotron radiation $\mu \mathrm{CT}$ data analyzed with digital volume correlation. J Struct Biol 2008;164(3):255-62.

[13] Réthoré J, Simon P, Maigre H. Multiscale digital image identification of heterogeneous elastic properties of softwoods. EPJ Web Conf 2010;6:18002.

[14] Gamstedt EK, Bader TK, de Borst K. Mixed numerical-experimental methods in wood micromechanics. Wood Sci Technol 2013;47:183-202.

[15] Eder M, Arnould O, Dunlop JWC, Hornatowska J, Salmén L. Experimental micromechanical characterisation of wood cell walls. Wood Sci Technol 2013;47:163-82

[16] Jäger A, Bader T, Hofstetter K, Eberhardsteiner J. The relation between indentation modulus, microfibril angle, and elastic properties of wood cell wall. Compos Part A: Appl Sci Manuf 2011;42:677-85.

[17] Johnson KL. Contact mechanics. Cambridge: Cambridge University Press; 1985.

[18] Jakes JE, Frihart CR, Beecher JF, Moon RJ, Resto PJ, Melgarejo ZH, et al. Nanoindentation near the edges. J Mater Res 2009;24(3):1016-31.

[19] Tranchida D, Piccarolo S. Local mechanical properties by atomic force microscopy nanoindentations. In: Bhushan B, Fuchs H, editors. Applied scanning probe methods, vol. XI. Berlin: Springer-Verlag; 2009. p. 165-98.

[20] Rabe U. Atomic force acoustic microscopy. In: Bhushan B, Fuchs H, editors. Applied scanning probe methods, vol. II. Berlin: Springer-Verlag; 2006. p. 37-90.

[21] Hurley DC. Contact resonance force microscopy techniques for nanomechanical measurements. In: Bhushan B, Fuchs H, editors. Applied scanning probe methods, vol. XI. Berlin: Springer-Verlag; 2009. p. 97-138.

[22] Clair B, Arinero R, Lévêque G, Ramonda M, Thibaut B. Imaging the mechanical properties of wood cell wall layers by atomic force modulation microscopy. IAWA 2004;24:223-30

[23] Bytebier $\mathrm{K}$, Arnould $\mathrm{O}$, Arinero $\mathrm{R}$. Mechanical characterization of wood viscoelasticity at the submicrometre scale. In: Proceedings of the COST FP0802 workshop on experimental and computational methods in wood micromechanics. Vienna; May 2009.

[24] Nair SS, Wang S, Hurley DC. Nanoscale characterization of natural fibers and their composites using contact-resonance force microscopy. Compos Part A: Appl Sci Manuf 2010;41:624-31.

[25] Newman GR. Use and abuse of LR White. Histochem J 1987;19:118-20.

[26]

Chang S, Quignard F, Di Renzo F, Clair B. Solvent polarity and internal stresses

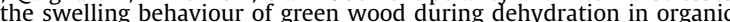
solution. BioResources 2012:7(2):2418-30.

[27] Kim J-W, Harper DP, Taylor AM. Technical note: effect of epoxy embedment on micromechanical properties of brown-rot-decayed wood cell walls assessed with nanoindentation. Wood Fiber Sci 2012;44(1):103-7.

[28] Konnerth J, Harper D, Lee SH, Rials TG, Gindl W. Adhesive penetration of wood cell walls investigated by scanning thermal microscopy (SThM). Holzforschung 2008;62(1):91-8.

[29] Gierlinger N, Hansmann C, Röder T, Sixta H, Gindl W, Wimmer R. Comparison of UV and confocal Raman microscopy to measure the melamineformaldehyde resin content within cell walls of impregnated spruce wood. Holzforschung 2005;59:210-3.

[30] Clair B, Gril J, Baba K, Thibaut B, Sugiyama J. Precautions for the structural analysis of the gelatinous layer in tension wood. IAWA 2005;26(2): 189-95.

[31] Jakes JE, Frihart CR, Beecher JF, Moon RJ, Stone DS. Experimental method to account for structural compliance in nanoindentation measurements. J Mater Res $2008 ; 23(4): 1113-27$.

[32] Arinero R, Lévêque G. Vibration of the cantilever in force modulation microscopy analysis by a finite element model. Rev Sci Instrum 2003;74: 10411

[33] Mège F, Volpi F, Verdier M. Mapping of elastic modulus at sub-micrometer scale with acoustic contact resonance AFM. Microelectron Eng 2010;87: 416-20.

[34] Arinero R, Lévêque G, Girard P, Ferrandis JY. Image processing for resonance frequency mapping in atomic force microscopy. Rev Sci Instrum 2007;78: 023703.

[35] Barthel E. Adhesive elastic contacts: JKR and more. J Phys D: Appl Phys 2008;41:163001. 
[36] Jakob AM, Buchwald J, Rauschenbacha B, Mayr SG. Nanoscale-resolved elasticity: contact mechanics for quantitative contact resonance atomic force microscopy. Nanoscale 2014;6:6898.

[37] Hurley DC, Kopycinska-Müller M, Julthongpiput D, Fasolka MJ. Influence of surface energy and relative humidity on AFM nanomechanical contact stiffness. Appl Surf Sci 2006;253:1274-81.

[38] Tung RC, Killgore JP, Hurley DC. Hydrodynamic corrections to contact resonance atomic force microscopy measurements of viscoelastic loss tangent. Rev Sci Instrum 2013;84:073703.

[39] Gindl W, Schöberlb T. The significance of the elastic modulus of wood cell walls obtained from nanoindentation measurements. Compos Part A: Appl Sci Manuf 2004;35:1345-9.

[40] Jäger A, Hofstetter K, Buksnowitz C, Gindl-Altmutter W, Konnerth J. Identification of stiffness tensor components of wood cell walls by means of nanoindentation. Compos Part A: Appl Sci Manuf 2011;42:2101-9.

[41] Socoliuc A, Gnecco E, Maier S, Pfeiffer O, Baratoff A, Bennewitz R, et al. Atomic-scale control of friction by actuation of nanometer-sized contacts. Science 2006;313:20710.

[42] TGT-01 by NT-MDT ${ }^{\mathrm{m} x}$ Test grating intended for 3-D visualization of the scanning tip. <http://www.ntmdt-tips.com/products/view/tgt1>.

[43] Delafargue A, Ulm FJ. Explicit approximations of the indentation modulus of elastically orthotropic solids for conical indenters. Int J Solids Struct 2004;41:7351-60.

[44] Le Rouzic J, Delobelle P, Vairac P, Cretin B. Comparison of three different scales techniques for the dynamic mechanical characterization of two polymers (PDMS and SU8). Euro Phys J - Appl Phys 2009;48:11201.

[45] Marinello F, Schiavuta P, Vezzù S, Patelli A, Carmignato S, Savio E. Atomic force acoustic microscopy for quantitative nanomechanical characterization. Wear 2011;271:534-8.

[46] Hurley DC. Quantitative measurements of elastic properties with ultrasonic-based AFM and conventional techniques. In: Marinello F, Passeri D, Savio E, editors. Acoustic Scanning Probe Microscopy. Berlin: Springer-Verlag; 2013. p. 351-73 [chapter $12]$.

[47] Killgore JP, Yablon DG, Tsou AH, Gannepalli A, Yuya PA, Turner JA, et al. Viscoelastic property mapping with contact resonance force microscopy. Langmuir 2011;27:13983-7.

[48] Killgore JP, Geiss RH, Hurley DC. Continuous measurement of atomic force microscope tip wear by contact resonance force microscopy. Small 2011;7(8):1018-22.

[49] Hanley SJ, Gray DG. Atomic force microscope images of black spruce wood sections and pulp fibres. Holzforschung 1994;48:29-34.
[50] Iliescu D, Géhin D, Nouari M, Girot F. Damage modes of the aeronautic multidirectional carbon/epoxy composite T300/914 in machining. Int J Mater Prod Technol 2008;32(2/3):118-1135.

[51] Donaldson L, Xu P. Microfibril orientation across the secondary cell wall of radiata pine tracheids. Trees 2005;19:644-53.

[52] Atkins AG, Vincent JFV. An instrumented microtome for improved histological sections and the measurement of fracture toughness. J Mater Sci Lett 1984;3:310-2.

[53] Zimmermann T, Thommen V, Reimann P, Hug HJ. Ultrastructural appearance of embedded and polished wood cell walls as revealed by atomic force microscopy. J Struct Biol 2006;156(2):363-9.

[54] Konnerth J, Gierlinger N, Keckes J, Gindl W. Actual versus apparent within cell wall variability of nanoindentation results from wood cell walls related to cellulose microfibril angle. J Mater Sci 2009;44:4399-406.

[55] Gierlinger N, Luss S, König C, Konnerth J, Eder M, Fratzl P. Cellulose microfibril orientation of Picea abies and its variability at the micron-level determined by Raman imaging. J Exp Bot 2010;61:587-95.

[56] Killgore JP, Hurley DC. Low-force AFM nanomechanics with higher-eigenmode contact resonance spectroscopy. Nanotechnology 2012;23:055702.

[57] Rodriguez BJ, Callahan C, Kalinin SV, Proksch R. Dual-frequency resonancetracking atomic force microscopy. Nanotechnology 2007;18:475504.

[58] Gannepalli A, Yablon DG, Tsou AH, Proksch R. Mapping nanoscale elasticity and dissipation using dual frequency contact resonance AFM. Nanotechnology 2011;22:355705.

[59] Cuberes MT, Stegemann B, Kaiser B, Rademann K. Ultrasonic force microscopy on strained antimony nanoparticles. Ultramicroscopy 2007;107:1053-60.

[60] Hurley DC, Turner JA. Measurement of Poisson's ratio with contact-resonance atomic force microscopy. J Appl Phys 2007;102:033509.

[61] Le Rouzic J, Delobelle P, Cretin B, Vairac P, Amiot F. Simultaneous measurement of Young's modulus and Poisson's ratio at microscale with two-modes scanning microdeformation microscopy. Mater Lett 2012;68:370-3. 